of those of their contemporaries. Mr. Harrisson's vivid and racy story of his personal experiences among unadministered natives on Malekula and Espiritu Santo serves as a foil, and redresses the balance as against the traditional view.

A tribute is paid to the medical work of presentday missions. The tendency of the population at the present time to increase is attributed in large measure to improvement in hygiene and medical attention, although the latter is still inadequate. The view of the late Dr. W. H. R. Rivers that the decline in population among the islanders of the Pacific was psychological in origin, and due to a tcedium vitce caused by the break-up of native custom, is regarded as disproved, at least so far as concerns the New Hebrides.

As a picture of island life seen in intimate conditions, Mr. Harrisson's book is a notable, as well as an entertaining, addition to the travel literature of the Pacific.

\title{
A New Higher Algebra
}

\section{Higher Algebra}

By S. Barnard and J. M. Child. Pp. xiv +585 . (London: Macmillan and Co., Ltd., 1936.) $20 \mathrm{~s}$.

$\mathrm{T}$ "HIS new "Higher Algebra" will be examined with great interest by all teachers and serious students of mathematics. A book of this type is certainly needed at the present time, and the one before us should go far towards providing that stimulus which the teaching of algebra urgently requires.

The book is rather an outsize, containing 585 pages, and at a first glance the reader is inclined to wonder why. For, in the main, the scope of the book is similar in extent to that of the older higher algebra texts; yet more than the usual amount of previous knowledge of mathematics is assumed : a "knowledge of the progressions and permutations and combinations". The authors might have added that the binomial theorem for a positive integral power is also assumed.

A closer examination shows that the various topics are treated very fully indeed, and that there are, in addition, several chapters which do not appear in the usual algebra text. For example, the early part of Chapter $v$, which deals with complex numbers, leads to the statement and development of De Moivre's theorem, the real and complex factors of $x^{n} \pm 1$ and vectors. Similarly, the discussion of continuity leads naturally to the derivative of a function and to the notation and methods of the calculus.

It is apparent that the authors have attached considerable importance to placing the notion of real number on a firm and sound basis; in this connexion, the book compares very favourably with many of its predecessors. On this sure foundation the subject is "developed logically, complete as far as it goes and serving as an introduction to modern analysis". Indeed, the title might well have been "An Introduction to Mathematical Analysis".

The arrangement of the work differs from that adopted in most higher algebra texts in that only as much of a topic is introduced as is immediately necessary for the development of the subject. For example, the theory of equations is not confined to a single chapter-the topic is introduced so early as Chapter vi and is further developed in Chapter xviii ; similarly with theory of numbers, polynomials, convergency, continued fractions, etc. In this way there is no suggestion of watertight compartments. Most of the topics are treated in considerable detail ; this is particularly the case in the treatment of the theory of numbers and the theory of equations.

Although a mathematically minded student might work through the book unaided (and so many hints are embodied in the exercises one feels that the authors have not forgotten this class of student) it is not suitable and is not intended for beginners. The Higher School Certificate students mentioned in the preface are students taking the mathematics group-and such students are supposed to be of special mathematical ability.

Both the publishers and the authors are to be congratulated on the finished appearance of the book; the printing is excellent, though some rather small type has been used in the worked examples, and the book has an appearance of solidity which is in keeping with its contents. There are many good sets of examples. Although the book is rather large, one feels that a few historical notes would have made for even more completeness than there is already. No teacher or serious student of mathematics should be without a copy of this excellent book. 